2010 to 2015. Importantly, our last-line treatment (ceftriaxone) is used in first-line dual therapy. However, over half of tested isolates are still sensitive to older drugs, e.g. ciprofloxacin. Discriminatory point-of-care tests (POCT) to detect drug sensitivity are under development, enabling individualised treatment decisions.

Methods We developed an individual-based transmission model of gonorrhoea infection in MSM, incorporating ciprofloxacinsensitive and resistant strains. The cumulative contact network is captured by periodically restricting active connexions to reflect the transience of high degree contacts. We explored different strategies to improve treatment selection including a) discriminatory POCT, and b) selecting partner treatment based on index case susceptibility. Outcomes included population prevalence and percentage reduction in ceftriaxone doses. Additional sensitivity analyses simulated the impact of reducing delays in the patient pathways on gonorrhoea prevalence.

Results The flexible model structure enabled us to efficiently explore a large region of parameter space, and credibly simulate London gonorrhoea transmission dynamics - assuming 2\%-10\% prevalence and 10-50 daily diagnoses per 100,000 MSM. Initial simulations show that a) using POCT to detect ciprofloxacin sensitive infections resulted in a $66 \%$ decrease in ceftriaxone doses, and b) using index case sensitivity profile to direct treatment of partners could reduce ceftriaxone use by $25 \%$.

Conclusion POCT are likely to dramatically reduce reliance on ceftriaxone. In the meantime, we could use existing data more informatively. If lab turnaround times are fast enough, index case sensitivity profiles could be used to select effective treatments for partners. This new framework addresses limitations of previous models and provides a flexible platform for exploring control options for AMR gonorrhoea.

\section{P2.02 CLINICAL AND HISTOLOGICAL ASPECTS OF HPV- INDUCED LESION IN THE ORAL CAVITY OF A PATIENT GROUP FROM A PRIVATE UNIVERSITY OF RIO DE JANEIRO BETWEEN 1998 AND 2015}

${ }^{1}$ Alexandre Marques Paes Da Silva, Mauro Romero Leal Passos, ${ }^{3}$ Helena Lúcia Barroso dos Reis, ${ }^{4}$ Luana Lorena Silva Rodrigues, ${ }^{5}$ Alcina Frederica Nico, ${ }^{1}$ Dennis Ferreira De Carvalho. 'Universidade Veiga de Almeida, Brazil; ${ }^{2}$ Universidade Federal Fluminense, Brazil; ${ }^{3}$ Universidade Federal do Espirito Santo, Brazil; ${ }^{4}$ Laboratório Interdisciplinar de Pesquisas Médicas - LIPMED-FIOCRUZ, Brazil; ${ }^{5}$ Instituto Oswaldo Cruz - LIPMED - IOC FIOCRUZ, Brazil

\subsection{6/sextrans-2017-053264.179}

Introduction Bearing in mind that the diagnosis of oral lesions, particularly those induced by HPV, may influence therapeutic method choice, the goal of this study was to describe the clinical and histological characteristics of these lesions in the oral cavity.

Methods A sectional laboratory research based on the reports obtained from the oral pathology laboratory of a private university of Rio de Janeiro, between 1998 to 2015 was held, with the objective of assessing the profile of 104 individuals diagnosed with HPV-induced oral lesions.
Results The lesions found were oral squamous papilloma (83\%), verruca vulgaris $(11,3 \%)$ and condyloma acuminata $(5,6 \%)$. The sites more commonly involved were the tongue (37 lesions, 33,9\%), the palate (21 lesions, 19,8\%) and lip mucosa (19 lesions, 17,9\%). The oral squamous papilloma was diagnosed in 87 patients, with average age of 40.3 years, race-colour white $(73 \%)$, being the tongue $(39,8 \%)$ and the palate $(22,7 \%)$ the more commonly described sites, with asymptomatic lesions of the type exophytic $(15,9 \%)$ and pedunculated $(13.6 \%)$ and had whitish colour, with an average size of about $0.55 \mathrm{~cm}$ in diameter. As for the 12 verruca vulgaris diagnosed, the individuals had an average age of 21.3 years, most of them affecting lip mucosa region with 7 lesions (to $58.3 \%$ ). The condyloma acuminata lesions were found in only 5 individuals, with ages ranging from 25 to 51 years of age, with an average of 32.8 years. The site of greatest occurrence was the lip mucous membrane with 3 lesions (50\%).

Conclusion In this study, it was possible to observe that women had more lesions in the oral cavity, being the tongue the most common site, and the oral squamous papilloma the most diagnosed lesion. The description of the histological and molecular aspects of these injuries becomes necessary so that there is an effective contribution to the knowledge of factors that may be associated with the development of these lesions in the oral cavity in the era of the quadrivalent/nonavalent vaccine.

\section{P2.03 ANTIBIOTIC TREATMENT FOR THE SEXUAL PARTNERS OF WOMEN WITH BACTERIAL VAGINOSIS}

J Amaya-Guio, CF Grillo-Ardila, DA Viveros-Carreño, EM Sierra-Barrios. Universidad Nacional de Colombia, Colombia

\subsection{6/sextrans-2017-053264.180}

Introduction Microorganisms associated with Bacterial Vaginosis (BV) have been isolated from the normal flora of the male genital tract, and their presence could be related to the recurrence of BV in women after antibiotic treatment. Therefore, the treatment of sexual partners could decrease the recurrence of infection.To assess the effectiveness in women and the safety in men of concurrent antibiotic treatment for the sexual partners of women treated for BV.

Search methods We searched the Cochrane STI Specialised Register, CENTRAL, MEDLINE, Embase, LILACS, International Clinical Trials Registry Platform, ClinicalTrials and Web of Science. We also handsearched conference proceedings.

Selection criteria Randomised controlled trials that compared the concurrent use of any antibiotic treatment with placebo by the sexual partners of women treated for BV.

Data collection and analysis Authors independently assessed trials for inclusion, extracted data and assessed the risk of bias. Disagreements were resolved through consensus. Quality of the evidence were assessed using GRADE.

Results Five trials (854 patients) met our inclusion criteria. High quality evidence shows that antibiotic treatment does not increase the rate of clinical or symptomatic improvement in women during the first week (risk ratio (RR) 0.99 , 95\% confidence interval (CI) 0.96 to 1.03 ; RR 1.06, 95\% CI 1.00 to 